\title{
Management of Term Breech Presentation and External Cephalic Version
}

\begin{abstract}
Summary
Previous studies have indicated that external cephalic version (ECV) has a high success rate and is safe in women who present with a breech presentation at term. The aim of this study was to examine the relationship between success rates and parity, mode of administration of tocolytic, maternal body mass index (BMI) and operator experience. Our study shows that there is no correlation between maternal BMI and success rates. Terbutaline administration and operator experience influence success rates when analysed using a logistic regression model, however multiparity is the strongest predictor of successful ECV. ECV is a safe procedure and should be offered to all women with breech presentation at term. A dedicated service with experienced operators should be available in tertiary units.
\end{abstract}

Keywords: External cephalic version, Operator skill, Parity, Spontaneous reversion, Tocolytic
Volume 7 Issue $3-2017$

\author{
Breffini C Anglim, Sean Daly,Aimee Cooper, \\ Gunther von Bunau \\ Coombe Women and Infant University Hospital, Ireland
}

Correspondence: Breffini C Anglim, Coombe Women and Infant University Hospital, Dublin, Ireland, Tel 00353 86I73455I, Email breffini.anglim@gmail.com

Received: October 25, 2016 | Published: July 05, 2017
Abbreviations: ECV, External Cephalic Version; BMI, Body Mass Index; CS, Caesarean Section

\section{Introduction}

Breech presentation occurs in 3 to $4 \%$ of all term pregnancies. Vaginal breech delivery rate has declined significantly since the Term Breech Trial, which showed lower serious perinatal mortality and neonatal morbidity after planned caesarean section delivery at term. ${ }^{1}$ ]. External cephalic version (ECV) is proven effective to prevent vaginal breech births and Caesarean sections. ${ }^{2}$ The fetus is manipulated from the breech to cephalic presentation through the maternal abdomen. Success rates between studies vary from $30-80 \%$. ECV is considered safe. There were no excess of intrauterine fetal deaths related to ECV recorded in a large series of 1,078 consecutive ECVs. ${ }^{3}$

Success rates are increased in women who are multiparous, flexed breeches, posterior placenta, anterior fetal back, non-engagement of the presenting part, palpable fetal head, amniotic fluid index of greater than $10 \mathrm{~cm}$, a thin maternal abdomen and an experienced operator. ${ }^{4-6}$

Race, uterine tone and the use of tocolytics also influence the success of ECV. The RCOG Guideline (Green-top 20a, March 2017) recommends that ECV should be offered from 36 weeks in nulliparous women and from 37 weeks in multiparous women. The RCOG further recommends a structured ECV service with preferably detailed written information about the procedure, its risks and benefits.

\section{Aim}

The aim of this study was to assess the success rates of external cephalic version (ECV) in a large tertiary maternity hospital in relation to parity, mode of tocolytic, maternal BMI and operator experience. Secondary aim was to observe the complication rate and assess the safety of the procedure.

\section{Methods}

This was a retrospective study which looked at data from January 2010 to January 2015. This was carried out in a tertiary referral centre with an annual delivery rate of between 9000 and 10,000 per year. Women with singleton pregnancies diagnosed with a breech presentation after 36 weeks gestation were given the option of ECV, and counselled appropriately, provided there were no contraindications to the procedure. Women who attended the Perinatal Centre for ECV were recorded in a dedicated ECV audit book. The study was approved by the Hospital Ethics Committee. Delivery outcome and maternal demographics were obtained from the hospital database and patient charts.

Elective caesarean section was offered at 39 weeks gestation in those women who declined ECV. Contraindications for ECV included previous uterine surgery (myomectomy, caesarean section), uterine anomalies, Doppler flow abnormalities, intrauterine growth restriction, fetal anomaly, prelabour rupture of membranes, advanced labour, antepartum haemorrhage in the third trimester and placenta praevia. Ultrasound was used prior to, during the procedure and following the procedure to determine success. Data was stored in a microsoft excel spreadsheet with data analysis being performed using DATADESK statistical software.

Factors which were studied to determine to association with ECV success rates included parity, BMI, mode of administration of tocolytic, operator seniority and spontaneous reversion rates after a successful ECV. Complications included antepartum haemorrhage, non-reassuring CTG, and the requirement for emergency caesarean section.

\section{Results}

There were no adverse perinatal outcomes during the study period and no cases of intrauterine death. However we had two cases, which we classified as complications, although there was no negative outcome. There was one case of antepartum haemorrhage which occurred four hours following a successful ECV which required observation and induction the following day. The source of the haemorrhage was not ascertained. There was no adverse perinatal outcome. One patient did not receive anti-D despite being Rhesus negative, but fortunately no antibodies developed when an indirect Coombs test was performed at 6 weeks postnatal.

A total of 325 women attended for a trial of ECV. Patient demographics are outlines in Table 1. The average age was 31.9(18-41). 
The mean BMI was 22.5 (17-49). The mean gestation at time of ECV was $37+4$ in nulliparae and $37+3$ in multiparae. The mean gestational age at delivery was $39+6(36+6-42+2)$. Overall the success rate was $50 \%$. The reversion rate following successful ECV was $6 \% .2 .5 \%$ of unsuccessful ECVs subsequently turned to a cephalic presentation prior to delivery. Seventy-eight percent of ECVs were carried out by obstetric consultants, with the remainder being performed by obstetric trainees, mainly under direct supervision and guidance. One consultant performed $32 \%$ of the total ECVs and another $14 \%$, with success rates of $63 \%$ and $70 \%$ respectively. A cardiotocograph was performed prior to and following each procedure and the woman's rhesus status was determined and anti-D given where indicated. The majority of women were given terbutaline $250 \mathrm{mcg}(97 \%)$ either intravenously (46\%) or subcutaneosly (51\%) prior to ECV.

Table I Characteristics of study population

\begin{tabular}{ll}
\hline Nulliparae & 177 \\
Multiparae & 148 \\
Para I & 74 \\
Para 2 & 42 \\
Para 3 & 22 \\
Para 4+ & 10 \\
Mean age & $31.9(18-4 I)$ \\
Mean BMI & $22.47(17-49)$ \\
Mean GA at ECV & $37+3(35+4-4 I)$ \\
Mean GA at delivery & $39+6(36+6-42+2)$ \\
\hline
\end{tabular}

There was a direct correlation between success rates and parity, with a $34 \%(61 / 177)$ success rate in nulliparae compared to $68 \%$ (100/148) in multiparae (58\% for Para 1, 80\% for Para 2 and $77 \%$ for Para 3+, see Graph 1). Women who were Para 2 or more were most likely to have a successful ECV $(\mathrm{P}=0.0008)$. Success rates were $52 \%, 65 \%, 59 \%, 63 \%$ and $80 \%$ at $36,37,38,39,40$ weeks gestation respectively, (Graph $2 \& 3$ ). There was no correlation between BMI and success rates $(\mathrm{P}=0.47)$, nor was there a correlation with morbid obesity, defined as a BMI $>40 \mathrm{~kg} / \mathrm{m} 2$ and success rates $(\mathrm{P}=0.3)$. Of the total number of ECVs, $78 \%$ of cases were performed by consultants. First consultant performed 105 and the other 44, with success rates of $63 \%$ and $70 \%$ respectively. The mode of delivery for successful and unsuccessful ECVs is outlined in Table 2.

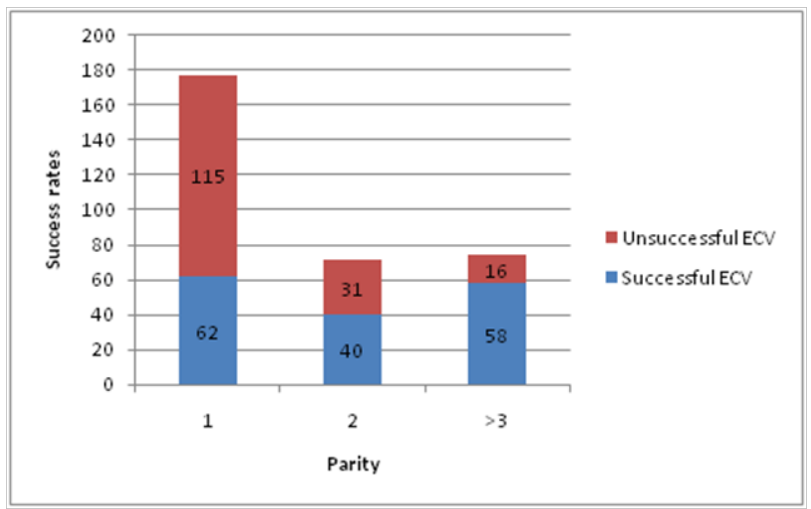

Graph I Relationship between success rates of ECV and parity.

With regard to tocolytic administration $46 \%$ had terbutaline $250 \mathrm{mcg}$ intravenously compared to $51 \%$ subcutaneously. $3 \%$ did not receive a tocolytic. The success rate of IV terbutaline was $58 \%$ compared to $45 \%$ in the SC group and $20 \%$ in those who did not receive terbutaline. There was a significant difference $(\mathrm{p}=0.02)$ between intravenous compared to subcutaneous administration.
Seventy-three percent of women successfully delivered vaginally post ECV. $86 \%$ of these were spontaneous vaginal deliveries, and the remainder instrumental deliveries.

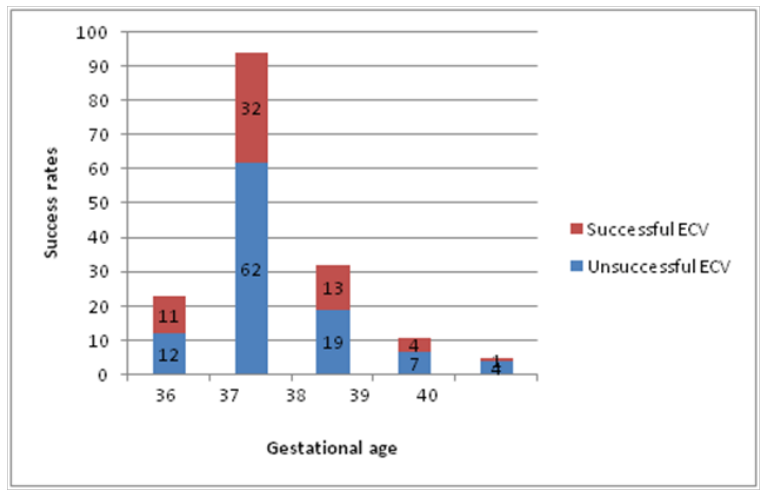

Graph 2 Relationship between success rates of ECV and gestational age in primiparous women.

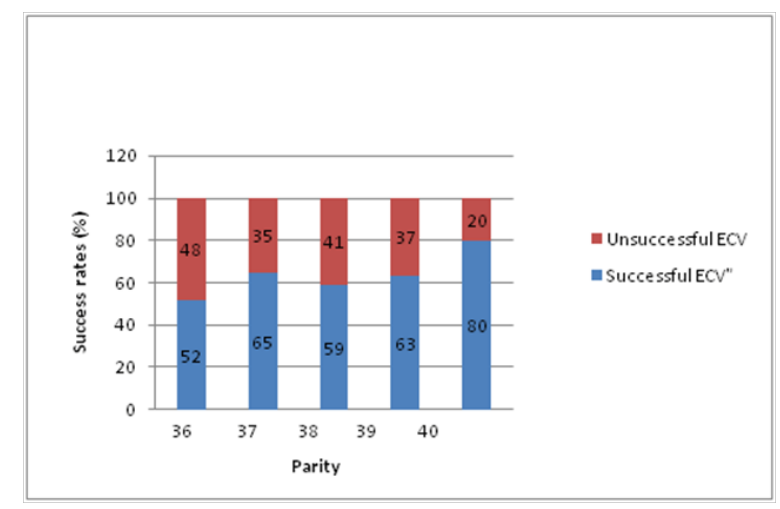

Graph 3 Relationship between success rates of ECV and gestational age in primiparous women.

Table 2 Mode of delivery after unsuccessful and successful ECV

\begin{tabular}{lll}
\hline Mode of delivery & Successful $(\mathbf{n})^{* *}$ & Unsuccessful $(\mathbf{n}=)^{*}$ \\
\hline Vaginal delivery & & 3 \\
SVD & 103 & 1 \\
Ventouse & 10 & 0 \\
Forceps & 6 & \\
Caesarean section & & 129 \\
Elective & 10 & 28 \\
Emergency & 34 &
\end{tabular}

*9 successful versions reverted

*2 successful version were an unstable lie

**3 turned to cephalic, of which 2 delivered spontaneously, I assisted delivery

A logistic regression model was performed for analysis. A logistic regression model with three variables including administration of terbutaline, parity and those consultants doing $>20 \mathrm{ECV}$ shows that all are associated with success but the strongest predictor is multiparity. There was a single complication involving a post-procedure vaginal bleed following an ECV procedure. This patient was induced the following day and had a successful spontaneous vaginal delivery without any perinatal morbidity

\section{Discussion}

ECV has proven effective in preventing vaginal breech births and Cesarean sections. The caesarean section rate was $27 \%$ during this period. Breech presentation accounted for $16 \%$ of caesarean deliveries. 
By performing ECVs we were able to reduce the Caesarean section rate by $12 \%$. Our data suggests that that there is a low uptake of ECV in women with breech presentation at term. We do not have figures for the number of women who were offered ECV and the number who declined. Interestingly, success rate of ECV was not correlated to BMI. This finding was not anticipated given the difficulty of palpation of fetal parts in women who are obese. However our study shows that ECV is a safe procedure, adding to previous studies in confirming that there is no associated excess in morbidity or mortality. There were no emergency $\mathrm{CS}$ following ECV, no admissions to the neonatal intensive care unit and intrauterine fetal deaths. ECV should therefore be offered to all women with breech presentation at term, if there are no contraindications.

A patient information leaflet and procedure checklist were developed. The procedure checklist would act to reduce the risk of failing to administer Anti-D in women who are rhesus negative and has been shown to be an effective method or reducing errors.

This study has strengths. BMI in our patient population was not self-reported, and was derived from maternal height and weight. This improves accuracy of BMI calculation significantly compared to self reported height and weight measurement. Performing an ECV like most obstetric procedures is more successful if the procedure has been performed by an experienced operator. Seniority of the operator was documented in all cases, something not present in other literature on this topic. Mode of delivery is available in all patients, which allows us to determine the percentage of women who despite successful ECV were delivered by emergency section, or those who reverted back to breech presentation.

\section{Conclusion}

Uptake of ECV may be improved with provision of leaflets and patient information. The outcome data from this study is useful for patient counseling and shows a high success rate for ECV. Ideally ECV should be done at 36 weeks gestation, with terbutaline administered intravenously by an experienced consultant. A dedicated ECV-Service is recommended and referral from smaller hospitals, who don't offer such a service should be considered.

\section{Acknowledgements}

This study describes original work and has not been submitted for consideration by any other journal. All authors have approved the manuscript and its submission. All authors have contributed substantially to obtaining and analyzing data obtained for this study. All authors have had input both drafting and revision of the article and final approval of the version published.

\section{Declaration of interest}

The authors report no conflicts of interest.

\section{References}

1. Hannah ME, Hannah WJ, Hewson SA, et al. Planned caesarean section versus planned vaginal birth for breech presentation at term: a randomised multicentre trial. Term Breech Trial Collaborative Group. Lancet. 2000;356(9239):1375-1383.

2. Vlemmix F, Rosman AN, Fleuren MA, et al. Implementation of the external cephalic version in breech delivery. Dutch national implementation study of external cephalic version. BMC Pregnancy Childbirth. 2010;10:20.

3. Leung T, Suen S, Sahota D, et al. Maternal, Fetal and Neonatal Medicine Sep. 2012;25(9):1774-1778.

4. Cho L, Lau W, Lo T, et al. Predictors of successful outcomes after external cephalic version in singleton breech pregnancies: a nine year historical cohort study. Hong Kong Med J. 2012118(1):11-19.

5. Arif W, Maqsood F, Iqbal M, et al. Prediction model for determining important factors of success of external cephalic version. J Ayub Med Coll Abbottabad. 2012;24(2):14-17.

6. Kok M, Cnossen J, Gravendeel L, et al. Clinical factors to predict the outcome of external cephalic version: a metaanalysis. Am J Obstet Gynecol. 2008;199(6):630.e1-630.e7.

7. Winters B, Gurses A, Lehmann H, et al. Clinical review: Checklists translating evidence into practice. Critical Care. 2009;13(6):210. 